\title{
$\underline{\mathbf{P}-135}$
}

\section{The Optimized Extraction Solvent System for Crude Extract of Stevia Rebaudiana in Detecting the Stevioside Presence}

\author{
Siti Safura Jaapar*, Syafiqah Zainoddin and Saliha Azlan \\ Faculty of Pharmacy, Universiti Teknologi MARA, $42300 \quad$ Puncak Alam, Malaysia; \\ E-mail: sitisa2790@puncakalam.uitm.edu.my
}

Stevia rebaudiana is an important sugar substitute belongs to Asteraceae family. The sweet taste of the species is contributed by stevioside, a natural sweetener extracted from the plant. This study is performed to detect the presence of stevioside in parts of $S$. rebaudiana plant and to determine the best solvent system in crude extraction of $S$. rebaudiana. Chemical extracts from dried shoots, leaves, stems and roots were attempted using three different extraction solvents which were hexane, methanol and water. Isolated stevioside was purified and analyzed by using Thin Layer Chromatography (TLC) method. The $\mathrm{R}_{\mathrm{f}}$ value obtained from $50 \%$ concentration of flowers, leaves, roots and shoots samples which were extracted with water were 0.41 , 0.44 , 0.41 and 0.41 respectively. While the $R_{f}$ value of $25 \%$ concentration of flowers, leaves, roots and shoots samples which were extracted with water were $0.35,0.38,0.39$ and 0.28 respectively. From the results, we can conclude that stevioside is present in flowers, leaves, roots and shoots of $S$. rebaudiana plant and water is the best extraction solvent in stevioside extraction process.

Keywords: Stevioside, Stevia rebaudiana, Extraction, TLC, Rf value. 